\title{
Thromboaspiration in a Patient With Persisting Intracranial Occlusion After Intravenous Thrombolysis: A Case Report
}

\author{
Fabrizio Sallustio a, b, d, Silvia Di Legge ${ }^{\mathrm{a}, \mathrm{b}}$, Domenico Sama, ${ }^{\mathrm{a}, \mathrm{b}}$, Giacomo Kocha, \\ Matteo Stefaninic, Costantino Del Giudicec, Enrico Pampanac, \\ Roberto Gandinic, Paolo Stanzione ${ }^{\mathrm{a}, \mathrm{b}}$, Giovanni Simonettic
}

\begin{abstract}
The last few years have been characterized by a rapid diffusion of endovascular treatments aimed at achieving arterial recanalization in patients with acute ischemic stroke. Clinical trials have shown higher rates of arterial recanalization being achieved by the Penumbra System (PS) as compared to standard treatment, i.e. systemic thrombolysis by recombinant tissue plasminogen activator (rtPA). This case-report enhances the relevance of strict patient monitoring during and after rtPA administration in order to consider urgent mechanical thrombectomy as additional treatment in highly selected cases. A 69-year-old woman presented with sudden onset of left hemiparesis and slurred speech. Systemic rtPA was administered with marked clinical improvement. Ultrasound study performed during rtPA infusion suggested intracranial occlusion. Diffusionweighted MRI was negative for acute ischemia, perfusion-weighted imaging detected a wide region of hypoperfused tissue in the right middle cerebral artery (MCA) territory, and MR angiography detected right proximal MCA occlusion. After the initial clinical improvement, the patient deteriorated. Clot aspiration by the PS and complete recanalization were achieved in 56 minutes. The patient was discharged with mild left hemiparesis, and she had excellent recovery at 6 months. Acute endovascular treatment by the PS proved successful in this patient experiencing clinical fluctuations due to
\end{abstract}

Manuscript accepted for publication February 14, 2012

${ }^{a}$ The Stroke Unit, Department of Neuroscience, University of Rome "Tor Vergata", Viale Oxford 8100133 Rome, Italy

bIRCCS Santa Lucia, Viale Ardeatina 30600134 Rome, Italy

'Department of Diagnostic and Molecular Imaging, Interventional Radiology and Radiation Therapy, University of Rome "Tor Vergata", Viale Oxford 8100133 Rome, Italy

${ }^{\mathrm{d} C}$ Corresponding author: Fabrizio Sallustio, Stroke Unit, Department of Neuroscience, University of Rome "Tor Vergata", Viale Oxford 81, 00133 Rome, Italy. Email: fabriziosallustio@libero.it

doi:10.4021/jnr84w persisting intracranial artery occlusion after systemic thrombolysis. Strict patient monitoring by MRI and ultrasound studies may allow prompt mobilization of the interventional radiology team thus offering an increased chance of favourable outcome and preventing more invalidating strokes in patients known to benefit poorly by systemic thrombolysis.

Keywords: Ischemic stroke; Intracranial artery occlusion; Thrombolysis; Endovascular treatment; Penumbra system

\section{Introduction}

The PS is a neurothrombectomy device indicated for the treatment of ischemic stroke caused by large vessel intracranial occlusion. The Penumbra Pivotal Stroke Trial included patients who presented within 8 hours of symptom onset and had an angiographically verified occlusion of a large intracranial vessel [1]. Patients who presented within 3 hours were either not eligible or refractory to intravenous (IV) administration of recombinant tissue plasminogen activator (rtPA) as defined by residual significant neurological deficits or persistent occlusion in the target vessel. Compared to rtPA treatment alone, the PS was associated with higher rate of arterial recanalization and reduced risk of haemorrhagic transformation [2]. Pending the results of randomized-controlled trials, this case-report underlines the possibility to adequately treat selected stroke patients in highly specialized centres.

\section{Case Report}

A 69-year-old woman with history of hypertension and paroxysmal atrial fibrillation was admitted in a peripheral hospital for sudden onset (at 2:45 pm) of left sensory-motor deficit, left facial droop and slurred speech with a NIH Stroke Scale (NIHSS) of 8. Head CT scan (3:44 pm) ruled out any intracranial haemorrhage and showed hyperdensity of the right middle cerebral artery (MCA). The patient was admitted in our stroke unit at 5:00, and the IV rtPA infusion was started at 5:30 pm (165 minutes after stroke onset). At 

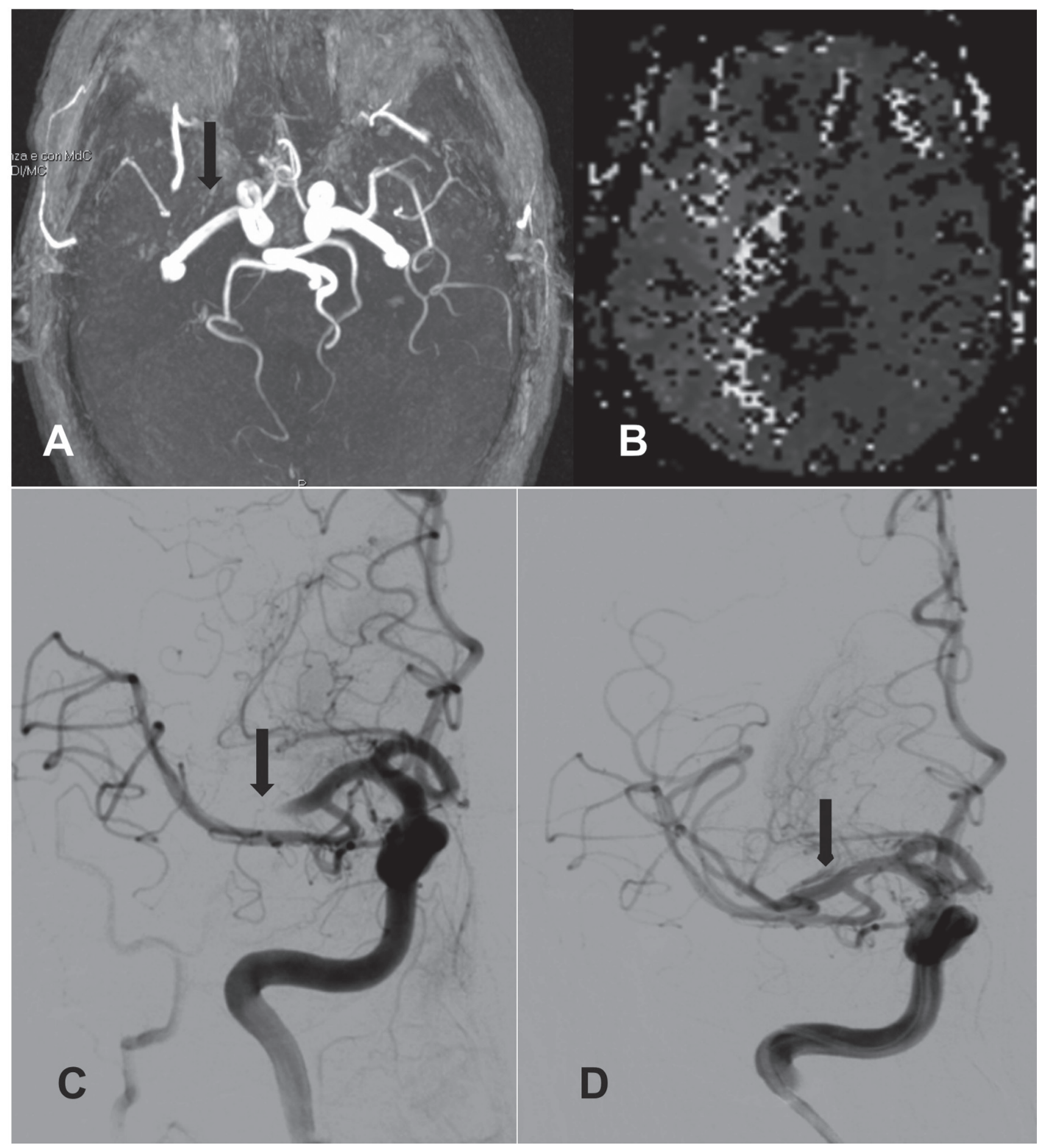

Figure 1: Figure 1. MRI and angiographic findings. Panel A: Time-of-flight MR angiography showing occlusion of proximal right middle cerebral artery (MCA) (black arrow); panel B: PW-MRI detects the hypoperfusion deficit in the right MCA territory; panel C: digital subtraction angiography (DSA) before thromboaspiration, confirming MCA occlusion (black arrow); panel D: DSA showing complete MCA recanalization after thromboaspiration.

the end of the infusion $(6: 30 \mathrm{pm})$ the NIHSS was 2, transcranial color-coded duplex sonography (TCCS) couldn't be performed because of insufficient temporal bone windows. However, an extracranial color-coded duplex sonography (ECCS) performed after the end of IV rtPA infusion detected high pulsatility index (PI) values in the right internal carotid artery (ICA), which was highly suggestive for ipsilateral intracranial arterial occlusion. Due to these findings, the interventional radiologist on call was alerted for a possible endovascular procedure. At 7:00 pm an MRI and MRA study showed no lesions on diffusion-weighted imaging (DWI), a wide region of hypoperfusion in the right MCA territory on mean transit time (MTT) and time-to-peak (TTP) perfusion weighted images (PWI) maps, and right M1 occlusion. On T2 FLAIR sequences hyperintense small cortical vessels were visualised in the right hemisphere. At the end of the MRI study (7:30 pm) the patient neurological conditions deteriorated (NIHSS $=13$ ), and urgent traditional angiography confirmed the presence of MCA occlusion. Clot aspiration by the PS with complete recanalization of the occluded vessel (TIMI 3) was achieved in 56 minutes. Figure 1 shows MRI findings and crucial phases of the endovascular proce- 
dure. The patient was discharged after five days on warfarin treatment (INR 2-3) for secondary stroke prevention in a patient with atrial fibrillation. At six-month follow-up clinic visit the patient had an NIHSS of 1, with a modified Rankin Scale $(\mathrm{mRS})=0$.

\section{Discussion}

We described a patient with early clinical worsening after IV rtPA administration due to persisting MCA occlusion, who was successfully treated by the PS. In a single-centre IV rtPA series, persisting MCA occlusion was observed in $22 \%$ of patients, and this group also had the lowest rate (8\%) of three good outcome compared to patients showing MCA recanalization $(50 \%)$ or vessel reocclusion (33\%) [3]. The study also showed that persisting MCA occlusion was related to clinical deterioration despite IV rtPA treatment.

In our patient, persistent MCA occlusion was suggested by ECCS findings and confirmed by brain MRI and MRA soon after IV thrombolysis. Hyperintense cortical vessels on FLAIR sequences has been shown to be a marker of activation of leptomeningeal collateral flow [4], could explain the clinical improvement observed after IV thrombolysis and represents a condition predicting favourable outcome in patients undergoing endovascular procedures [5]. Further, a wide perfusion deficit on PWI associated with an MCA occlusion suggested an unstable haemodynamic condition despite the patient initial clinical improvement. A rapid intervention could be performed because the endovascular team had been previously mobilized in light of combined CT, ECCS, MRI and MRA findings.

In our institution the PS is currently used, in highly selected cases, as adjunctive treatment after IV thrombolysis within 8 hours from symptom onset. Considering the Penumbra Pivotal Stroke Trial [1], our patient didn't fall within the existing inclusion criteria since she has initial favourable response (not refractory) to IV thrombolysis with an NIHSS of 2 excluding indication for any further acute treatment. After clinical worsening, while further pharmacological strategies could not be considered due to the previous recent use of rtPA, the availability of an endovascular procedure proved essential. Thus, this anecdotal case underlines the key role of performing DWI/PWI MRI study after IV rtPA administration. This, in turn, allows hemodynamic monitoring and planning of additional early interventions aimed at increasing the change of good recovery, also preventing more invalidating strokes.

\section{Disclosures}

None.

\section{References}

1. The penumbra pivotal stroke trial: safety and effectiveness of a new generation of mechanical devices for clot removal in intracranial large vessel occlusive disease. Stroke. 2009;40(8):2761-2768.

2. Baker WL, Colby JA, Tongbram V, Talati R, Silverman IE, White CM, Kluger J, et al. Neurothrombectomy devices for the treatment of acute ischemic stroke: state of the evidence. Ann Intern Med. 2011;154(4):243-252.

3. Alexandrov AV, Grotta JC. Arterial reocclusion in stroke patients treated with intravenous tissue plasminogen activator. Neurology. 2002;59(6):862-867.

4. Liebeskind DS. Collateral circulation. Stroke. 2003;34(9):2279-2284.

5. Bang OY, Saver JL, Kim SJ, Kim GM, Chung CS, Ovbiagele B, Lee $\mathrm{KH}$, et al. Collateral flow predicts response to endovascular therapy for acute ischemic stroke. Stroke. 2011;42(3):693-699. 